Acta Univ. Sapientiae Informatica 12, 2 (2020) 217-231

DOI: 10.2478 /ausi-2020-0013

\title{
Degree tolerant coloring of graph
}

\author{
Johan KOK \\ Independent Mathematics Researcher, City of \\ Tshwane, South Africa \& Visiting Faculty at \\ CHRIST (Deemed to be a University), Bangalore, \\ India \\ email: jacotype@gmail.com
}

\begin{abstract}
This paper initiates a study on a new coloring regime which sets conditions in respect of the degrees $\operatorname{deg}(v)$ and $\operatorname{deg}(u)$ where, $v, u \in$ $\mathrm{V}(\mathrm{G})$ and $v \mathrm{u} \in \mathrm{E}(\mathrm{G})$. This new coloring regime is called, "degree tolerant coloring". The degree tolerant chromatic number is defined. A number of interesting introductory results are presented. Amongst others, new Nordhaus-Gaddum type bounds are provided.
\end{abstract}

\section{Introduction}

For general notation and concepts in graphs see $[2,5,8]$. Throughout only finite, undirected, simple graphs will be considered. It is assumed that the reader is familiar with the concept of graph coloring. Recall that in a proper coloring of $\mathrm{G}$ all edges are good i.e. $\forall \mathfrak{u} v \in E(G), c(u) \neq c(v)$. The set of colors assigned in a proper graph coloring is denoted by $\mathcal{C}$ and a subset of colors assigned to a subset of vertices $\mathrm{X} \subseteq \mathrm{V}(\mathrm{G})$ is denoted by $\mathrm{c}(\mathrm{X})$. In an improper (or defect) coloring it is permitted that for some $u v \in E(G)$, the coloring is $\mathrm{c}(\mathrm{u})=\mathrm{c}(\boldsymbol{v})$. It is evident that improper coloring has been formally defined and studied by $[3,4,6]$.

Since any graph $\mathrm{G}$ has the parameters, $\delta(\mathrm{G})$ and $\Delta(\mathrm{G})$, an integer degree condition related to an integer $k, \delta(G) \leq k \leq \Delta(G)$ will be introduced. For

Computing Classification System 1998: G.2.2

Mathematics Subject Classification 2010: 05C15, 05C38, 05C75, 05C85

Key words and phrases: Degree tolerant coloring, degree tolerant chromatic number, proper coloring, defect coloring 
a "degree tolerant coloring" abbreviated as, DT-coloring of a graph G the following conditions are set:

(i) If $u v \notin E(G)$ then, either $c(u)=c(v)$ or $c(u) \neq c(v)$;

(ii) If $u v \in E(G)$ and $\operatorname{deg}(u)=\operatorname{deg}(v)$ then, $c(u)=c(v)$ else, $c(u) \neq c(v)$.

Alternative formulation for condition (ii). If $u v \in E(G)$ then, $c(u)=c(v)$ if and only if $\operatorname{deg}(u)=\operatorname{deg}(v)$.

The motivation for this study is that, it is fundamentally acceptable to "do mathematics for the sake of mathematics". From an application point of view the vertices could represent communication elements (graph vertices in graph context) in a communication network. Failure of a communication vertex $u$ could be replaced by a neighbor $v$ if and only $\operatorname{deg}(u)=\operatorname{deg}(v)$. This can be made possible by imbedding equivalent or identical technology in both $u$ and $v$. The technology equivalence is characterised by equal degrees for such pair of neighbors. The aforesaid could be viewed as spontaneous merging of such vertices on failure of any one vertex. Hence, a maximum number of such merging operations exists for a communication network before complete communication failure occurs. Analogy is found in electrical networks where one network relies on say, conventional fossil fuel generation and another relies of solar generation which is then distributed via an inverter plant. On failure of either, the neighbor can provide electricity to the other through a switch over protocol. The author foresees that interesting informatica research can result from this introductory paper.

The minimum number of colors which yields a DT-coloring is called the degree tolerant chromatic number of $\mathrm{G}$ and is denoted by, $\mathrm{\chi}_{\mathrm{dt}}(\mathrm{G})$. For certain classes of connected graphs the value of $\chi_{\mathrm{dt}}(\mathrm{G})$ follows immediately. For example, for paths we have $\chi_{d t}\left(P_{1}\right)=\chi_{d t}\left(P_{2}\right)=1$ and $\chi_{d t}\left(P_{n}\right)=2, n \geq 3$. For all cycles, $\chi_{d t}\left(C_{n}\right)=1, n \geq 3$. All regular graphs $G$ have $\chi_{d t}(G)=1$. Also, the null graph (edgeless) $\mathfrak{N}_{n}$ has $\chi_{\mathrm{dt}}\left(\mathfrak{N}_{\mathfrak{n}}\right)=1$. Clearly there exist graphs for which, $\chi_{\mathrm{dt}}(\mathrm{G}) \leq \chi(\mathrm{G})$. However, it is easy to verify that the graph $\mathrm{G}$ in Figure 1 has, $\chi_{\mathrm{dt}}(\mathrm{G})>\chi(G)$.

As an introductory paper a variety as aspects are considered. Section 2 deals with preliminaries on general results and with elementary graph operations. Section 3 deals with three standard graph products. In addition, new Nordhaus-Gaddum type bounds are provided. In the conclusion some further research avenues are mentioned. 


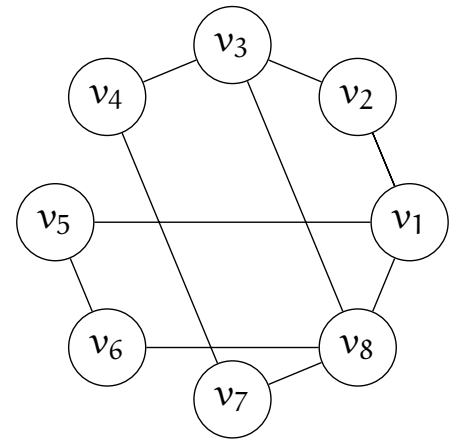

Figure 1: Graph $G$ for which $\chi(G)=2$ and $\chi_{\mathrm{dt}}(\mathrm{G})=3$

\section{Some general results}

In the literature a wide range of coloring regimes have been defined and many have been well researched. It is rare to find a formal result to show that all graphs permit a particular coloring regime. The aforesaid is mostly stated or silently assumed to be true. However, there does exist at least one coloring regime called, Johan coloring (or $\mathcal{J}$-coloring) which is not permitted by all graphs. See [7] and related references. It cannot be assumed that all graphs permit a DT-coloring but this is true in this case.

Theorem 1 Any graph permits a DT-coloring.

Proof. We proof the result through induction on $n$. At first, assume the graph $\mathrm{G}$ is a connected graph of order $n$. It is known that $\chi_{\mathrm{dt}}\left(K_{1}\right)=1$ hence, the result holds a connected graphs of order 1 . Also, $\chi_{d t}\left(P_{2}\right)=1$ hence, the result holds for all connected graphs of order 2 . For $n=3$ we have two cases to consider.

Case $1_{3}$. Let $G=P_{3}$. It is known that, $\chi_{d t}\left(P_{3}\right)=2$.

Case $2_{3}$. Let $G=K_{3}$. Since $K_{3}$ is a regular graph, $\chi_{d t}\left(K_{3}\right)=1$.

Thus far the result holds for all connected graphs of order 3 .

For connected graphs of order 4, six cases up to isomorphism must be considered.

Case $1_{4}$. For $\mathrm{P}_{4}$ we have, $\chi_{\mathrm{dt}}\left(\mathrm{P}_{4}\right)=2$.

Case $2_{4}$. For the star $S_{1,3}$ it easily follows that, $\chi_{\mathrm{dt}}\left(S_{1,3}\right)=2$.

Case 34 . For the graph $G$ obtained from $K_{3}$ on vertices $v_{1}, v_{2}, v_{3}$ with a single pendant vertex $u$ attached to any vertex of $K_{3}$ say, to $v_{1}$ the coloring, $\mathrm{c}\left(v_{1}\right)=\mathrm{c}_{1}, \mathrm{c}\left(v_{2}\right)=\mathrm{c}\left(v_{3}\right)=\mathrm{c}(\mathrm{u})=\mathrm{c}_{2}$ is a permissible DT-coloring. 
Case $4_{4}$. For the graph $\mathrm{C}_{4}$ a DT-coloring is permissible because $\mathrm{C}_{4}$ is regular.

Case $5_{4}$. For the graph $\mathrm{G}$ obtained from $\mathrm{C}_{4}$ on vertices $v_{1}, v_{2}, v_{3}, v_{4}$ with the addition of a chord say, $v_{1} v_{3}$, the coloring $\mathrm{c}\left(v_{1}\right)=\mathrm{c}\left(v_{3}\right)=\mathrm{c}_{1}$ and $\mathrm{c}\left(v_{2}\right)=$ $\mathrm{c}\left(v_{4}\right)=\mathrm{c}_{2}$ is a permissible DT-coloring.

Case $6_{4}$. It is known that $\mathrm{K}_{4}$ permits a DT-coloring.

The basis for the induction assumption is now well established. Assume the result holds for all connected graphs on $1 \leq \mathrm{q} \leq \mathrm{n}$ vertices. Let the distinct connected graphs of order $n$ up to isomorphism be, $G_{1}, G_{2}, G_{3}, \ldots, G_{k}$. Consider any connected graph $\mathrm{G}_{i}, 1 \leq i \leq \mathrm{k}$ on $\mathrm{q}=\mathrm{n}$ vertices. To reason for the case $q=n+1$ begin with the disconnected graph $G_{i} \cup K_{1}$. Let $V\left(K_{1}\right)=$ $\left\{v_{n+1}\right\}$. Also assume the color set $\mathcal{C}=\left\{c_{i}: i=1,2,3, \ldots, k\right\}$ is a DT-coloring set of $G_{i}$.

Construct $\mathrm{G}_{i}^{\prime}$ by adding any number of edges say, $t$ edges, $1 \leq t \leq n$ given by,

$$
X=\underbrace{\left\{v_{\mathrm{n}+1} v_{\mathrm{i}}, v_{\mathrm{n}+1} v_{\mathrm{j}}, v_{\mathrm{n}+1} v_{\mathrm{k}}, \ldots, v_{\mathrm{n}+1} v_{\mathrm{s}}\right\}}_{(\mathrm{t} \text { edges })}
$$

Clearly, $\operatorname{deg}\left(v_{n+1}\right)=t$. Also the open neighborhood of $v_{n+1}$ is, $N\left(v_{n+1}\right)=X=$ $\left\{v_{i}, v_{j}, v_{k}, \ldots, v_{s}\right\}$.

If $\operatorname{deg}\left(v_{n+1}\right) \neq \operatorname{deg}\left(v_{i}\right), v_{i} \in X$ then let $\mathrm{c}\left(v_{n+1}\right)$ be any color in $\mathcal{C} \backslash \mathrm{c}(X)$ if possible else, assign a new color say $\mathrm{c}^{*}$. In both cases, be it $\mathcal{C}$ or $\mathcal{C} \cup\left\{\mathrm{c}^{*}\right\}$ assigned, a minimum DT-coloring is obtained.

If $\operatorname{deg}\left(v_{n+1}\right)=\operatorname{deg}\left(v_{i}\right)$, for some $v_{i} \in X$ we have the following subcases.

(i) If $\operatorname{deg}\left(v_{n+1}\right)=\operatorname{deg}\left(v_{i}\right)$, for exactly one $v_{i} \in X$, let $c\left(v_{n+1}\right)=c\left(v_{i}\right)$ to yield a permissible DT-coloring of $\mathrm{G}_{i}^{\prime}$.

(ii) Let $\operatorname{deg}\left(v_{n+1}\right)=\operatorname{deg}\left(v_{i}\right)$, for two or more vertices in X. If the said two or more adjacent vertices have identical coloring say, color $c_{r}$ then assign $c\left(v_{n+1}\right)=c_{r}$. If at least one of the said adjacent vertices has a coloring other than the rest then recolor to $\mathrm{c}\left(X \cup\left\{v_{n+1}\right\}\right)$ any color in $\mathcal{C}$ and through iterative neighborhood re-assigned coloring obtain a DT-coloring, $\varphi: \mathrm{V}\left(\mathrm{G}_{\mathfrak{i}}^{\prime}\right) \mapsto \mathcal{C}$ if possible. Otherwise, assign $c\left(X \cup\left\{v_{n+1}\right\}\right)=c^{*}$. Therefore, $\mathcal{C} \cup\left\{c^{*}\right\}$ is a permissible DT-coloring of $\mathrm{G}_{i}^{\prime}$.

Through immediate induction the results holds for all connected graphs $\mathrm{G}_{i}^{\prime}$, $1 \leq i \leq k$. Put differently, it holds for all connected graphs of order $n+1$. Hence, for all connected graphs of order $\mathrm{q}, 1 \leq \mathrm{q} \leq \mathrm{n}+1$. Therefore, through mathematical induction it hold for all connected graphs of finite order.

Assume the graph $\mathrm{H}$ has $\mathrm{t}$ connected components i.e. $\mathrm{H}_{1}, \mathrm{H}_{2}, \mathrm{H}_{3}, \ldots, \mathrm{H}_{\mathrm{t}}$. Clearly, $\chi_{d t}(H)=\max \left\{\chi_{d t}\left(H_{i}\right)\right.$ : for some $\left.i, 1 \leq i \leq t\right\}$. Since all $H_{i}$ permit a 
DT-coloring it follows that $\mathrm{H}$ permits a DT-coloring. Hence, the result holds for any simple graph of order $n, n \in \mathbb{N}$.

Remark: The proof of Theorem 1 can be achieved through a reconstructive technique. Recall that the formal definition of a graph $G$ of order $n$ is, an ordered triple $\left(V(G), E(G), l_{G}\right)$ consisting of an non-empty set $V(G)$ of vertices say, $\left\{v_{1}, v_{2}, v_{3}, \ldots, v_{n}\right\}$ and a set $E(G)$, disjoint from $V(G)$, of edges and an incidence function, $\mathfrak{l}_{\mathrm{G}}$ that associates with each edge of $\mathrm{G}$ an unordered pair of vertices of $\mathrm{G}$ (vertices in an unordered pair are not necessarily distinct). Begin with the null graph (edgeless) denoted by, $\mathfrak{N}_{n}$ on the vertices $\left\{v_{1}, v_{2}, v_{3}, \ldots, v_{n}\right\}$. Note that $\chi_{\mathrm{dt}}\left(\mathfrak{N}_{n}\right)=1$. Let $\mathrm{E}(\mathrm{G})=\left\{e_{1}, e_{2}, e_{3}, \ldots, e_{q}\right\}$. Reconstruct $G$ by iteratively adding the edges $e_{i}, i=1,2,3, \ldots, q$ and by assigning a DT-coloring iteratively. Observe that after adding the edge $e_{1}$ the result, $\chi_{\mathrm{dt}}\left(\mathfrak{N}_{n}+e_{1}\right)=$ 1 follows. If edge $e_{2}$ is added such that $e_{1}, e_{2}$ are incident then the result, $\chi_{\mathrm{dt}}\left(\left(\mathfrak{N}_{n}+e_{1}\right)+e_{2}\right)=2$ follows. Else, $\chi_{\mathrm{dt}}\left(\left(\mathfrak{N}_{n}+e_{1}\right)+e_{2}\right)=1$. The observation is that $\mathrm{G}$ can be reconstructed iteratively and after each iteration a DT-coloring can be assigned. Formalising this approach as a proof is left to the reader.

Henceforth, only simple connected graphs will be considered. We now present some general results for $\chi_{d t}(G)$. Note that $\chi_{d t}\left(K_{n}\right)=1$ because $K_{n}$ is a regular graph.

Theorem 2 For $\mathrm{n} \in \mathbb{N}$ there exists a graph $\mathrm{G}$ with, $\chi_{\mathrm{dt}}(\mathrm{G})=\mathrm{n}$.

Proof. Obviously, $\chi_{\mathrm{dt}}\left(\mathrm{K}_{1}\right)=1$. Also, $\chi_{\mathrm{dt}}\left(\mathrm{P}_{3}\right)=2$. For $n \geq 3$ begin with the complete graph $K_{n}$. Let $V\left(K_{n}\right)=\left\{v_{1}, v_{2}, v_{3}, \ldots, v_{n}\right\}$. Construct a new graph $G$ by attaching to each vertex $v_{i}$ an arbitrary number $k_{i} \geq 0$ pendent vertices such that, $k_{i} \neq k_{j}$ if and only if $i \neq j$. Clearly $d_{G}\left(v_{i}\right) \neq d_{G}\left(v_{j}\right)$ if $i \neq j$. However, $v_{i} v_{j} \in \mathrm{V}(\mathrm{G})$ hence, $\mathrm{c}\left(v_{\mathrm{i}}\right) \neq \mathrm{c}\left(v_{\mathrm{j}}\right)$. Therefore, $\chi_{\mathrm{dt}}(\mathrm{G}) \geq \mathrm{n}$. Without loss of generality let the pendent vertices adjacent to $v_{i}$ be labeled $u_{i, \ell}, 1 \leq \ell \leq k_{i}$. Because $\mathrm{d}_{\mathrm{G}}\left(\mathrm{u}_{\mathrm{i}, \ell}\right)=1 \leq \mathrm{d}_{\mathrm{G}}\left(\nu_{\mathrm{i}}\right)$ and $\mathrm{u}_{\mathrm{i}, \ell} v_{\mathrm{i}} \in \mathrm{E}(\mathrm{G})$ the coloring $\mathrm{c}\left(\mathrm{u}_{\mathrm{i}, \ell}\right)=\mathrm{c}_{\mathrm{j}}$, $j \neq i$ is permissible. Thus $\chi_{d t}(G) \leq n$ and the result, $\chi_{d t}(G)=n$ follows.

Recall that the order and size (number of edges) of a graph is denoted by $v(G)$ and $\varepsilon(G)$. For a graph parameter $p(G)$ of specific value say, $k \in \mathbb{N}$ a minimal graph $G$ is a graph with $\min \{v(G)+\varepsilon(G)\}$ which yields, $p(G)=$ $k$. Observe that for $k=1$ the minimal graph $G=K_{1}$ yields, $\chi_{d t}(G)=1$. For $k=2$ the minimal graph $G=P_{3}$ yields, $\chi_{d t}(G)=2$. For $k=3$ the minimal graph is the dart graph. The dart graph has order 5 and size 6 . These observations can be verified exhaustively against the list of small graphs at, www.graphclasses.org/smallgraphs.html We claim that, to construct such minimal graph the founding or initial graph is the complete graph of order 
k. Firstly, we see that $\mathrm{K}_{1}, \mathrm{~K}_{2}\left(\right.$ or $\left.\mathrm{P}_{2}\right)$ and $\mathrm{K}_{3}\left(\right.$ or $\left.\mathrm{C}_{3}\right)$ are complete as well as regular graphs. Note that for $k=4$ one could reasonably consider to begin with the trivial founding graph $\mathrm{C}_{4}$ on vertices $v_{1}, v_{2}, v_{3}, v_{4}$, which is 2-regular. However, to achieve distinct colorings i.e. $c\left(v_{1}\right) \neq c\left(v_{j}\right)$ if $i \neq j$ with the minimal constructive additions of graph elements (vertices or edges), a $\mathrm{K}_{4}$ will inevitably result through any construction methodology. Inductive reasoning is the basis of our claim.

Theorem 3 For $\mathrm{k} \in \mathbb{N}$ there exists a minimal graph $\mathrm{G}$ of order $\mathrm{n}=2 \mathrm{k}-1$ $(\operatorname{or} v(\mathrm{G})=2 \mathrm{k}-1)$ and size $\mathcal{\varepsilon}(\mathrm{G})=\mathrm{k}(\mathrm{k}-1)$ for which, $\mathrm{\chi}_{\mathrm{dt}}(\mathrm{G})=\mathrm{k}$. Also, this minimal graph is unique.

Proof. Obviously for $k \in \mathbb{N}$ and $\chi_{\mathrm{dt}}(\mathrm{G})=\mathrm{k}$ we must have, $v(\mathrm{G}) \geq \mathrm{k}$. It follows that for $k=1$, the graph $K_{1}$ is minimal of order, $2 \times 1-1=1$.

For $k \geq 2$, begin with a graph putting all vertices on equal footing with regards to degree, i.e. having equal degree. Let the complete graph be on vertices $v_{1}, v_{2}, v_{3}, \ldots, v_{k}$. For $v_{i}, i=2,3,4, \ldots, k$ add a distinct pendent vertex $u_{i}$. Also add the edges $u_{i} v_{i+j}, i=2,3,4, \ldots,(k-1), j=1,2,3, \ldots,(k-i)$ to obtain G. Clearly, the aforesaid addition of pendent vertices and edges is the minimum constructive additions of graph elements to ensure, $\operatorname{deg}\left(v_{i}\right) \neq$ $\operatorname{deg}\left(v_{j}\right)$ for $i \neq j$. Clearly, from conditions (i) and (ii) the minimum color set $\mathcal{C}=\left\{c_{1}, c_{2}, c_{3}, \ldots, c_{k}\right\}$ is required to assign a DT-coloring to G. From the construction of $\mathrm{G}$ it follows that $\mathrm{G}$ is a minimal graph hence, $\min \{v(\mathrm{G})+\varepsilon(\mathrm{G})\}$, $\forall v, \varepsilon \in \mathbb{N}$ to yield, $\chi_{\mathrm{dt}}(\mathrm{G})=\mathrm{k}$. Furthermore, $v(\mathrm{G})=2 \mathrm{k}-1$ and $\varepsilon(\mathrm{G})=$ $\frac{k(k-1)}{2}+\frac{(k-1) k}{2}=k(k-1)$. Minimality and uniqueness of $G$ follow directly from the stringent and unambiguous construction methodology.

Figure 2 depicts the unique minimal graph for which $\chi_{\mathrm{dt}}(\mathrm{G})=3$. For example let, $c\left(v_{1}\right)=c_{1}, c\left(v_{2}\right)=c_{2}, c\left(v_{3}\right)=c_{2}, c\left(u_{2}\right)=c_{1}, c\left(u_{3}\right)=c_{1}$.

Theorem 3 leads to an important inverse result.

Theorem 4 For a graph $\mathrm{G}$ of order $\mathrm{n} \geq 1$ it follows that,

$$
\chi_{\mathrm{dt}}(\mathrm{G}) \leq\left\lfloor\frac{n+1}{2}\right\rfloor .
$$

Proof. Observe that for $k=1,2,3,4 \ldots$ Theorem 3 provides the minimum order of graphs i.e. $n=1,3,5,7, \ldots, 2 k-1, \ldots$ for which a minimal graph $G$ can be constructed such that, $\chi_{\mathrm{dt}}(\mathrm{G})=\mathrm{k}$. It follows that for any graph $\mathrm{G}^{\prime}$ of order $\ell, 2 k-1<\ell<2 k+1$ we have, $\chi_{\mathrm{dt}}\left(\mathrm{G}^{\prime}\right)<k+1$. Thus $\chi_{\mathrm{dt}}\left(\mathrm{G}^{\prime}\right) \leq k$.

Since for $k \in \mathbb{N}$, the minimal graph $G$ is of order $n=2 k-1$, it implies that:

$$
n-2 k+1=0 \text { has root at, } k=\frac{n+1}{2} \text {. }
$$




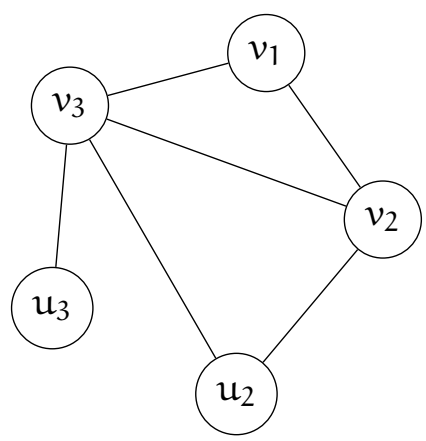

Figure 2: Unique minimal graph for which $\chi_{\mathrm{dt}}(\mathrm{G})=3$

For an integer solution it follows that,

$$
\chi_{\mathrm{dt}}(\mathrm{G}) \leq\left\lfloor\frac{n+1}{2}\right\rfloor .
$$

Theorem 5 For a graph $\mathrm{G}$ of size $\varepsilon(\mathrm{G})=\mathrm{q} \geq 1$ it follows that,

$$
\chi_{\mathrm{dt}}(\mathrm{G}) \leq\left\lfloor\frac{1+\sqrt{1+4 q}}{2}\right\rfloor \text {. }
$$

Proof. We know that for $k=1,2,3,4 \ldots$, Theorem 3 provides the minimum size of graphs i.e. $q=0,2,6,12, \ldots, k(k-1), \ldots$ for which a minimal graph $\mathrm{G}$ can be constructed such that, $\chi_{\mathrm{dt}}(\mathrm{G})=k$. It follows that for any graph $\mathrm{G}^{\prime}$ of size $\ell, k(k-1)<\ell<(k+1) k$ we have, $\chi_{\mathrm{dt}}\left(\mathrm{G}^{\prime}\right)<k+1$. Thus, $\chi_{\mathrm{dt}}\left(\mathrm{G}^{\prime}\right) \leq k$.

Since for $q \in \mathbb{N}$, the minimal graph $G$ is of size $q=k(k-1)$, it implies that:

$$
q-k(k-1)=0 \text { has roots at, } \frac{1 \pm \sqrt{1+4 q}}{2} \text {. }
$$

For an integer solution it follows that,

$$
\chi_{\mathrm{dt}}(\mathrm{G}) \leq\left\lfloor\frac{1+\sqrt{1+4 \mathrm{q}}}{2}\right\rfloor .
$$

For $n$ as determined by Theorem 3 in respect of $k \in \mathbb{N}$, let $C_{1}(G)$ be all graphs of order $n$.

Corollary 6 A graph $\mathrm{G} \in \mathrm{C}_{1}(\mathrm{G})$ has $\mathrm{\chi}_{\mathrm{dt}}(\mathrm{G}) \leq \mathrm{k}$.

Proof. The result is a direct consequence of Theorems 3 and 4 .

For $\mathrm{q}$ as determined by Theorem 3 in respect of $k \in \mathbb{N}$, let $C_{2}(G)$ be all graphs of of size $q$. 
Corollary 7 Araph $\mathrm{G} \in \mathrm{C}_{2}(\mathrm{G})$ has $\mathrm{\chi}_{\mathrm{dt}}(\mathrm{G}) \leq \mathrm{k}$.

Proof. The result is a direct consequence of Theorems 3 and 5 .

Corollary 8 A minimal graph $\mathrm{G}$ in respect of $\mathrm{k}$-degree tolerant coloring is always of odd order and even size.

A more significant result which follows directly from Theorems 4 and 5 is presented.

Theorem 9 For a graph $\mathrm{G}$ of order $\mathrm{n}$ and size $\mathrm{q}$ we have,

$$
\chi_{\mathrm{dt}}(\mathrm{G}) \leq \min \left\{\left\lfloor\frac{n+1}{2}\right\rfloor,\left\lfloor\frac{1+\sqrt{1+4 q}}{2}\right\rfloor\right\} .
$$

\subsection{On three elementary graph operations}

Consider non-trivial graphs $\mathrm{G}$ and $\mathrm{H}$ and the elementary graph operations known as the disjoint union $\mathrm{G} \cup \mathrm{H}$, the corona $\mathrm{G} \circ \mathrm{H}$ and the join $\mathrm{G}+\mathrm{H}$.

Proposition 10 For graphs $\mathrm{G}$ and $\mathrm{H}$ on order $\mathrm{n}$ and $\mathrm{m}$ respectively:

(a) $\chi_{\mathrm{dt}}(\mathrm{G} \cup \mathrm{H})=\max \left\{\chi_{\mathrm{dt}}(\mathrm{G}), \chi_{\mathrm{dt}}(\mathrm{H})\right\}$.

(b)

$$
\chi_{\mathrm{dt}}(\mathrm{G} \circ \mathrm{H})= \begin{cases}\chi_{\mathrm{dt}}(\mathrm{G}), & \text { if } \chi_{\mathrm{dt}}(\mathrm{G})>\chi_{\mathrm{dt}}(\mathrm{H}) \\ \chi_{\mathrm{dt}}(\mathrm{H})+1, & \text { if } \chi_{\mathrm{dt}}(\mathrm{G}) \leq \chi_{\mathrm{dt}}(\mathrm{H})\end{cases}
$$

\section{Proof.}

(a) Trivial.

(b) From a $\chi_{\mathrm{dt}}(\mathrm{G})$-color set $\mathcal{C}$ assign a DT-coloring to graph $\mathrm{G}$. In the graph $\mathrm{G} \circ \mathrm{H}$ a vertex $\mathrm{u}$ in a copy of $\mathrm{H}$ has, $\operatorname{deg}(\mathrm{u}) \leq \mathrm{m}$. In the graph $\mathrm{G} \circ \mathrm{H}$ a vertex $v$ in $G$ has, $\operatorname{deg}(v) \geq 1+\mathrm{m}$. Hence, $\mathrm{c}(v)$ is distinct from any vertex color in the copy of $\mathrm{H}$. For purposes of reasoning, begin by coloring each copy of $\mathrm{H}$ with its independent DT-coloring (ignoring adjacency of the corresponding vertex in $\mathrm{V}(\mathrm{G})$.)

Case 1: If $\chi_{d t}(G)>\chi_{d t}(H)$ and $c(v)=c_{i}$ then all vertices in $u \in V(H)$ for which $c(u)=c_{i}$, may be recolored with any color in the set $\mathcal{C} \backslash\left\{c_{i}\right\}$. The coloring obtained is a permissible DT-coloring of $\mathrm{G} \circ \mathrm{H}$. Hence, $\chi_{\mathrm{dt}}(\mathrm{G} \circ \mathrm{H})=\chi_{\mathrm{dt}}(\mathrm{G})$.

Case 2. If $\chi_{\mathrm{dt}}(\mathrm{G}) \leq \chi_{\mathrm{dt}}(\mathrm{H})$ and $c(v)=c_{i}$ then all vertices in $u \in V(H)$ for which $c(u)=c_{i}$, must be recolored with a new color $c_{\chi_{d t}(H)+1}$. Hence, $\chi_{d t}(G \circ H)=\chi_{d t}(H)+1$. 
Lemma 11 For a graph $\mathrm{G}$ partition $\mathrm{V}(\mathrm{G})$ into vertex subsets, $\mathrm{P}=\left\{\mathrm{X}_{1}, \mathrm{X}_{2}, \mathrm{X}_{3}\right.$, $\left.\ldots, X_{\mathrm{t}}\right\}$ such that $v_{j}, v_{\mathrm{k}} \in X_{\mathrm{i}}$ if and only if $\operatorname{deg}\left(v_{\mathrm{j}}\right)=\operatorname{deg}\left(v_{\mathrm{k}}\right)$ else, $\left|\mathrm{X}_{\mathrm{i}}\right|=1$. It follows that, $\mathrm{X}_{\mathrm{dt}}(\mathrm{G}) \leq|\mathrm{P}|$.

Proof. Let a set of distinct colors be $\mathcal{C}=\left\{c_{1}, c_{2}, c_{3}, \ldots, c_{|P|}\right\}$ together with the mapping, $c\left(X_{i}\right) \mapsto c_{i}, \forall i$. Assume that the partition coloring does not correspond to a $\chi_{\mathrm{dt}^{-}}$-coloring of $G$ and that $\chi_{\mathrm{dt}}(\mathrm{G})>|\mathrm{P}|$. It implies that for at least one $X_{i} \in P$ there exists at least one vertex $v_{j} \in X_{i}$. Therefore, $c\left(v_{j}\right)=c_{l}$, $c_{l} \notin \mathcal{C}$ is required. If $v_{j} v_{k} \in \mathrm{E}(\mathrm{G}), \mathrm{c}\left(v_{\mathrm{k}}\right)=\mathrm{c}_{\mathrm{k}} \in \mathcal{C}$, it is a contradiction in terms of condition (ii). Hence, $\mathrm{c}\left(v_{\mathrm{j}}\right)=\mathrm{c}_{\mathrm{i}}$ is permissible. If $v_{\mathrm{j}} v_{\mathrm{k}} \notin \mathrm{E}(\mathrm{G})$, then by condition (i) the coloring $\mathrm{c}\left(v_{j}\right)=\mathrm{c}_{\mathrm{i}}$ is permissible. Therefore, $\chi_{\mathrm{dt}}(\mathrm{G}) \leq|\mathrm{P}|$.

Corollary 12 For graph $\mathrm{G}$ the degree tolerant chromatic number is bounded by, $1 \leq \chi_{\mathrm{dt}}(\mathrm{G}) \leq|\mathrm{P}|$.

Association with vertex partition $\mathrm{P}$. Obviously $\mathrm{P}$ is a partition of the vertex set of a graph. The parameter, $\operatorname{deg}_{G}\left(X_{i}\right)=\operatorname{deg}_{G}(v), v \in X_{i}$ is also associated with $P$. Finally, for $P=\left\{X_{i}: 1 \leq i \leq t\right\}$ of $V(G)$ and $P^{\prime}=$ $\left\{Y_{1}, Y_{2}, Y_{3}, \ldots, Y_{l}\right\}$ of $V(H)$ we define the operation, $P \ominus P^{\prime}=k$, where $k$ is the number of vertex subsets, $Y_{j}$ with $\operatorname{deg}_{H}\left(Y_{j}\right) \neq \operatorname{deg}_{G}\left(X_{i}\right), \forall i$.

Reduction procedure: Reflecting on Lemma 11 it is observed that, if a vertex subset, $X_{i} \in P$ can itself be partitioned such that, $X_{i}=\bigcup_{j=1}^{r} X_{i, j}$ and for each $X_{i, j}$ there exists some vertex subset $X_{k} \in P, i \neq k$ such that, $\forall v_{l} \in X_{i, j}$ and $\forall v_{\mathrm{t}} \in X_{\mathrm{k}}$ the edge $v_{\mathrm{l}} v_{\mathrm{t}} \notin \mathrm{E}(\mathrm{G})$, then the upper bound can be decreased (improved) by 1 .

Example. Consider the path $\mathrm{P}_{3}$ on vertices $v_{1}, v_{2}, v_{3}$. Attach pendent vertices $\mathfrak{u}_{1}, \mathfrak{u}_{2}, \mathfrak{u}_{3}, \mathfrak{u}_{4}$ to $v_{1}$ to obtain the graph $G$. Utilising Lemma 11 the vertex partition, $P=\left\{\left\{v_{1}\right\},\left\{v_{2}\right\},\left\{v_{3}, u_{1}, u_{2}, u_{3}, u_{4}\right\}\right\}$ is obtained. Hence, $\chi_{\mathrm{dt}}(\mathrm{G}) \leq 3$. Note that the vertex subset $\left\{v_{3}, u_{1}, u_{2}, u_{3}, u_{4}\right\}$ itself can be partitioned into $\left\{\left\{v_{1}\right\},\left\{\mathfrak{u}_{1}, \mathfrak{u}_{2}, \mathfrak{u}_{3}, \mathfrak{u}_{4}\right\}\right\}$. Observe that edge, $v_{1} v_{3} \notin E(G)$ and edges, $\mathfrak{u}_{1} v_{2}, u_{2} v_{2}, u_{3} v_{2}$, $\mathrm{u}_{4} v_{2} \notin \mathrm{E}(\mathrm{G})$. Thus, $\chi_{\mathrm{dt}}(\mathrm{G}) \leq 2$. When the stated reduction procedure is exhausted, equality is attained. In the example, $\chi_{\mathrm{dt}}(\mathrm{G})=2$.

Proposition 13 For graphs $\mathrm{G}$ and $\mathrm{H}$ of order $\mathrm{n}$ and $\mathrm{m}$ respectively, we have: $\chi_{\mathrm{dt}}(\mathrm{G}+\mathrm{H})=\chi_{\mathrm{dt}}(\mathrm{G})+\left(\mathrm{P} \ominus \mathrm{P}^{\prime}\right)$.

Proof. Without loss of generality let $n \geq m$. Assign a DT-coloring to G. Such DT-coloring exists by Theorem 1 . Let the DT-color set be, $\mathcal{C}=\left\{c_{1}, c_{2}, c_{3}, \ldots, c_{t}\right\}$ 
and let $V(G)=\left\{v_{1}, v_{2}, v_{3}, \ldots, v_{n}\right\}$. Clearly, in the join $G+H$ the vertex degrees of $V(G)$ increases by the constant $m$ hence, $\operatorname{deg}_{G+H}\left(v_{i}\right)=\operatorname{deg}_{G}\left(v_{i}\right)+m$, $1 \leq i \leq n$. Therefore, the color set $\mathcal{C}$ remains a DT-color set for the induced subgraph $\langle V(G)\rangle$. Also, $V(G)$ can be partitioned into $P=\left\{X_{1}, X_{2}, X_{3}, \ldots, X_{t}\right\}$ such that, $c\left(X_{i}\right)=c_{i} \in \mathcal{C}, i=1,2,3, \ldots, t$.

Similarly, partition $V(H)=\left\{\mathfrak{u}_{1}, \mathfrak{u}_{2}, \mathfrak{u}_{3}, \ldots, \mathfrak{u}_{\mathrm{m}}\right\}$ in accordance to an assigned DT-coloring of $H$. Let this partition be $P^{\prime}=\left\{Y_{1}, Y_{2}, Y_{3}, \ldots, Y_{l}\right\}$. Note that each vertex degree in $\mathrm{V}(\mathrm{H})$ has increased with a constant i.e. $\operatorname{deg}_{\mathrm{G}+\mathrm{H}}\left(\mathrm{u}_{\mathrm{i}}\right)=$ $\operatorname{deg}_{G}\left(u_{i}\right)+n, 1 \leq i \leq m$. Clearly, the vertices of $V(H)$ in a partition subset, $Y_{i} \in P^{\prime}$ which has $\operatorname{deg}_{G+H}\left(Y_{i}\right)=\operatorname{deg}_{G+H}\left(X_{j}\right)$, must be colored $c\left(Y_{i}\right)=c\left(X_{j}\right)$. See condition(ii). Those which have $\operatorname{deg}_{G+H}\left(Y_{i}\right) \neq \operatorname{deg}_{G+H}\left(X_{j}\right), \forall i$ must be colored with a new color not in $\mathcal{C}$. See condition (ii). Hence, by Lemma 11, $\chi_{\mathrm{dt}}(\mathrm{G}+\mathrm{H}) \leq \chi_{\mathrm{dt}}(\mathrm{G})+\left(\mathrm{P} \ominus \mathrm{P}^{\prime}\right)$. Because DT-colorings were assigned to both $\mathrm{G}$ and $\mathrm{H}$ the reasoning of Lemma 11 has been met. Also, the reduction procedure has implicitly been exhausted. Therefore, $\chi_{d t}(G+H)=\chi_{d t}(G)+\left(P \ominus P^{\prime}\right)$.

Due to the commutative property of $G+H$ it follows that, $\chi_{d t}(G+H)=$ $\chi_{\mathrm{dt}}(\mathrm{G})+\left(\mathrm{P} \ominus \mathrm{P}^{\prime}\right)=\chi_{\mathrm{dt}}(\mathrm{H})+\left(\mathrm{P}^{\prime} \ominus \mathrm{P}\right)$.

\section{$3 \quad$ On graph products}

Consider two graphs $\mathrm{G}$ and $\mathrm{H}$ of order $\mathrm{n}$ and $\mathrm{m}$, respectively. Let the respective vertex sets be, $V(G)=\left\{v_{1}, v_{2}, v_{3}, \ldots, v_{n}\right\}$ and $V(H)=\left\{u_{1}, u_{2}, u_{3}, \ldots, u_{m}\right\}$. Recall that in general, a graph product is defined on the vertices $V(G) \times V(H)$. Adjacency (symbolised by, $\sim$ ) in the graph product is defined by conditions of adjacency (or non-adjacency) or equality, between pairs of distinct vertices in $\mathrm{V}(\mathrm{G})$ and/or $\mathrm{V}(\mathrm{H})$. It is known that $2^{8}=256$ products can be defined. This section will address three standard graph products. Note that in the literature there are different names corresponding to some of these graph products.

Convention: It is agreed that a comparable copy of a graph $\mathrm{G}$ means, comparable diagrammatic presentation including self evident comparable vertex labeling. For example, if the path $\mathrm{P}_{3}$ is sketch horizontally with the vertices labeled from left to right as, $v_{1}, v_{2}, v_{3}$ then, a comparable copy (just copy for brevity) could be on the vertices labeled from left to right as, $v_{i}, v_{i+1}, v_{i+2}$, $i \geq 4$. It also implies that $v_{1}$ corresponds to $v_{i}$ and it does not correspond to, $v_{i+2}$.

(i) Recall that adjacency in the Cartesian product denoted by, $\mathrm{G} \square \mathrm{H}$ is defined by $\left(v_{i}, u_{j}\right) \sim\left(v_{k}, u_{t}\right)$ if, $v_{i}=v_{k}$ and $u_{j} \sim u_{t}$ or, $v_{i} \sim v_{k}$ and $u_{j}=u_{t}$. To visualize this adjacency definition, simply replace each vertex of $G$ with 
a copy of $\mathrm{H}$. For the vertex $v_{i} \in \mathrm{V}(\mathrm{G})$ label such copy, $\mathrm{H}_{v_{i}}$. If the edge $v_{i} v_{j}$ exists then add an edge between corresponding vertices of the copies, $\mathrm{H}_{v_{i}}$ and $\mathrm{H}_{v_{j}}$.

(ii) Recall that adjacency in the tensor product (also called, categorical product) denoted by, $\mathrm{G} \times \mathrm{H}$ is defined by $v_{\mathrm{i}} \sim v_{\mathrm{k}}$ and $\mathrm{u}_{\mathrm{j}} \sim \mathrm{u}_{\mathrm{t}}$. To visualize this adjacency definition is not easy. What is important to note is that, if the respective degree sequences are,

$$
\left(\operatorname{deg}\left(v_{1}\right), \operatorname{deg}\left(v_{2}\right), \operatorname{deg}\left(v_{3}\right), \ldots, \operatorname{deg}\left(v_{n}\right)\right)
$$

and,

$$
\left(\operatorname{deg}\left(u_{1}\right), \operatorname{deg}\left(u_{2}\right), \operatorname{deg}\left(u_{3}\right), \ldots, \operatorname{deg}\left(u_{m}\right)\right)
$$

then die degree sequence of $\mathrm{G} \times \mathrm{H}$ is given by, ( $\operatorname{deg}\left(v_{1}\right) \operatorname{deg}\left(u_{1}\right), \operatorname{deg}\left(v_{1}\right) \operatorname{deg}\left(u_{2}\right), \operatorname{deg}\left(v_{1}\right) \operatorname{deg}\left(u_{3}\right), \ldots, \operatorname{deg}\left(v_{1}\right) \operatorname{deg}\left(u_{m}\right)$, $\operatorname{deg}\left(v_{2}\right) \operatorname{deg}\left(u_{1}\right), \operatorname{deg}\left(v_{2}\right) \operatorname{deg}\left(u_{2}\right), \operatorname{deg}\left(v_{2}\right) \operatorname{deg}\left(u_{3}\right), \ldots, \operatorname{deg}\left(v_{2}\right) \operatorname{deg}\left(u_{m}\right)$, ...

$\cdots$

...

$\left.\operatorname{deg}\left(v_{n}\right) \operatorname{deg}\left(u_{1}\right), \operatorname{deg}\left(v_{n}\right) \operatorname{deg}\left(u_{2}\right), \operatorname{deg}\left(v_{n}\right) \operatorname{deg}\left(u_{3}\right), \ldots, \operatorname{deg}\left(v_{n}\right) \operatorname{deg}\left(u_{m}\right)\right)$.

(iii) Recall that adjacency in the lexicographical product denoted by, $\mathrm{G} \bullet \mathrm{H}$ is defined by $v_{i} \sim v_{k}$ or, $v_{i}=v_{k}$ and $u_{j} \sim u_{t}$. To visualize this adjacency definition, simply replace each vertex of $G$ with a copy of $H$. For the vertex $v_{i} \in \mathrm{V}(\mathrm{G})$ label such copy, $\mathrm{H}_{v_{i}}$. If the edge $v_{i} v_{j}$ exists then add all edges of the join, $\mathrm{H}_{v_{\mathrm{i}}}+\mathrm{H}_{v_{j}}$.

Definition $14 \operatorname{Let}\left(\operatorname{deg}\left(v_{1}\right), \operatorname{deg}\left(v_{2}\right), \operatorname{deg}\left(v_{3}\right), \ldots, \operatorname{deg}\left(v_{n}\right)\right)$ be the degree sequence of graph $\mathrm{G}$. The degree index of graph $\mathrm{G}$ denoted by $\operatorname{di}(\mathrm{G})$, is the number of distinct vertex degree values.

Note that for any path $\left.P_{n}\right), n \geq 3$ we have, $\operatorname{di}\left(P_{n}\right)=2$. For any cycle $C_{n}$, $n \geq 3$ we have, $\operatorname{di}\left(C_{n}\right)=1$.

Theorem 15 For graphs $\mathrm{G}$ and $\mathrm{H}$ it follows that,

$$
\chi_{\mathrm{dt}}(\mathrm{G} \square \mathrm{H})=\min \left\{\operatorname{di}(\mathrm{H}) \chi_{\mathrm{dt}}(\mathrm{G}), \operatorname{di}(\mathrm{G}) \chi_{\mathrm{dt}}(\mathrm{H})\right\} .
$$

Proof. It is known that the Cartesian product is commutative to isomorphism. Hence, $\mathrm{G} \square \mathrm{H} \cong \mathrm{H} \square \mathrm{G}$.

Case 1. Consider $\mathrm{G} \square \mathrm{H}$. Replace each vertex $v_{i} \in \mathrm{V}(\mathrm{G})$ with $\mathrm{H}_{v_{i}}$. The adjacency condition, $v_{i}=v_{k}$ and $u_{j} \sim u_{t}$ is immediately satisfied. Do the following 
in respect of each $\left(v_{i}, u_{j}\right)_{j=1,2,3, \ldots, m}, i=1,2,3, \ldots, n-1$. For each neighbor of $v_{i}$ in $G$ add an edge between the vertices $\left(v_{i}, u_{j}\right), j=1,2,3, \ldots, m$ and between the respective corresponding vertices in the neighboring copy of $\mathrm{H}$, if such edges do not exist. After the stated procedure the condition $v_{\mathrm{i}} \sim v_{\mathrm{k}}$ and $u_{j}=u_{t}$ has been satisfied. Hence, $G \square H$ has been obtained. Clearly, $\operatorname{deg}\left(\left(v_{i}, u_{j}\right)\right)=\operatorname{deg}\left(u_{j}\right)+\left|N\left(v_{i}\right)\right|$. Following from Lemma 11, $\chi_{\mathrm{dt}}(\mathrm{G} \square \mathrm{H}) \leq$ $\operatorname{di}(\mathrm{G}) \chi_{\mathrm{dt}}(\mathrm{H})$.

Case 2. Consider $\mathrm{H} \square \mathrm{G}$. By similar reasoning as in Case 1 it follows that, $\chi_{\mathrm{dt}}(\mathrm{H} \square \mathrm{G}) \leq \operatorname{di}(\mathrm{H}) \chi_{\mathrm{dt}}(\mathrm{G})$.

Thus far by Lemma 11, we showed that,

$$
\chi_{\mathrm{dt}}(\mathrm{G} \square \mathrm{H}) \leq \min \left\{\operatorname{di}(\mathrm{H}) \chi_{\mathrm{dt}}(\mathrm{G}), \operatorname{di}(\mathrm{G}) \chi_{\mathrm{dt}}(\mathrm{H})\right\} .
$$

Since the reduction procedure has implicitly been exhausted for at least one of the two cases it follows that,

$$
\chi_{\mathrm{dt}}(\mathrm{G} \square \mathrm{H})=\min \left\{\operatorname{di}(\mathrm{H}) \chi_{\mathrm{dt}}(\mathrm{G}), \operatorname{di}(\mathrm{G}) \chi_{\mathrm{dt}}(\mathrm{H})\right\} .
$$

Theorem 16 For graphs $\mathrm{G}$ and $\mathrm{H}$ it follows that, $\chi_{\mathrm{dt}}(\mathrm{G} \times \mathrm{H}) \leq \operatorname{di}(\mathrm{G} \times \mathrm{H})$.

Proof. It is known that the tensor product is commutative to isomorphism. Hence, $\mathrm{G} \times \mathrm{H} \cong \mathrm{H} \times \mathrm{G}$.

The degree sequence of $\mathrm{G} \times \mathrm{H}$ is given by: $\left(\operatorname{deg}\left(v_{1}\right) \operatorname{deg}\left(u_{1}\right), \operatorname{deg}\left(v_{1}\right) \operatorname{deg}\left(u_{2}\right), \operatorname{deg}\left(v_{1}\right) \operatorname{deg}\left(u_{3}\right), \ldots, \operatorname{deg}\left(v_{1}\right) \operatorname{deg}\left(u_{m}\right)\right.$, $\operatorname{deg}\left(v_{2}\right) \operatorname{deg}\left(u_{1}\right), \operatorname{deg}\left(v_{2}\right) \operatorname{deg}\left(u_{2}\right), \operatorname{deg}\left(v_{2}\right) \operatorname{deg}\left(u_{3}\right), \ldots, \operatorname{deg}\left(v_{2}\right) \operatorname{deg}\left(u_{m}\right)$,

$\cdots$

$\cdots$

...

$\left.\operatorname{deg}\left(v_{n}\right) \operatorname{deg}\left(u_{1}\right), \operatorname{deg}\left(v_{n}\right) \operatorname{deg}\left(u_{2}\right), \operatorname{deg}\left(v_{n}\right) \operatorname{deg}\left(u_{3}\right), \ldots, \operatorname{deg}\left(v_{n}\right) \operatorname{deg}\left(u_{m}\right)\right)$.

Hence, the minimum number of colors needed to be assigned is $\operatorname{di}(\mathrm{G} \times \mathrm{H})$. The reduction procedure can be used to yield equality on a case by case basis. Therefore, $\chi_{\mathrm{dt}}(\mathrm{G} \times \mathrm{H}) \leq \operatorname{di}(\mathrm{G} \times \mathrm{H})$.

Theorem 17 For graphs $\mathrm{G}$ and $\mathrm{H}$ it follows that, $\mathrm{\chi}_{\mathrm{dt}}(\mathrm{G} \bullet \mathrm{H}) \leq \operatorname{di}(\mathrm{G}) \mathrm{\chi}_{\mathrm{dt}}(\mathrm{H})$.

Proof. It is known that the lexicographical product is associative but not commutative. Therefore, the reasoning is similar to that stated in the proof of Theorem 15, Case 1. However, exhaustion of the reduction procedure is not self-evident. Hence, $\chi_{\mathrm{dt}}(\mathrm{G} \bullet \mathrm{H}) \leq \operatorname{di}(\mathrm{G}) \chi_{\mathrm{dt}}(\mathrm{H})$. 


\subsection{Nordhaus-Gaddum type bounds}

Relations between a graph $\mathrm{G}$ and its complement $\overline{\mathrm{G}}$ have been studied since the inception of graph theory. Perhaps the most interesting category of relations between the two graphs are those with regards to the sum and products of graph parameters. The first known such relations were introduced by Nordhaus and Gaddum in 1956. The relations provide lower and upper bounds on the sum and the product of the chromatic number of a graph and its compliment. A comprehensive survey of the wide field which developed over the years can be found in [1].

Definition 14 read together with Lemma 11 imply that for a graph G, $\operatorname{di}(\mathrm{G})=$ t. Also, $\operatorname{deg}_{\bar{G}}\left(v_{i}\right)=(n-1)-\operatorname{deg}_{G}\left(v_{i}\right) \Rightarrow \operatorname{di}(\bar{G})=\operatorname{di}(G)$.

Theorem 18 For a graph $\mathrm{G}$ of order $\mathrm{n}$ and size $\mathrm{q}$ it holds that,

(a)

$$
\begin{aligned}
2 & \leq \chi_{\mathrm{dt}}(\mathrm{G})+\chi_{\mathrm{dt}}(\overline{\mathrm{G}}) \leq 2 \mathrm{di}(\mathrm{G}), \\
1 & \leq \chi_{\mathrm{dt}}(\mathrm{G}) \cdot \chi_{\mathrm{dt}}(\overline{\mathrm{G}}) \leq \mathrm{di}(\mathrm{G})^{2} .
\end{aligned}
$$

Weaker bounds are;

(b)

$$
\begin{gathered}
2 \leq \chi_{\mathrm{dt}}(\mathrm{G})+\chi_{\mathrm{dt}}(\overline{\mathrm{G}}) \leq 2\left(\left\lfloor\frac{n+1}{2}\right\rfloor\right), \\
1 \leq \chi_{\mathrm{dt}}(\mathrm{G}) \cdot \chi_{\mathrm{dt}}(\overline{\mathrm{G}}) \leq\left\lfloor\frac{n+1}{2}\right\rfloor^{2} .
\end{gathered}
$$

(c)

$$
\begin{aligned}
& 2 \leq \chi_{\mathrm{dt}}(\mathrm{G})+\chi_{\mathrm{dt}}(\overline{\mathrm{G}}) \leq\left\lfloor\frac{1+\sqrt{1+4 \mathrm{q}}}{2}\right\rfloor+\left\lfloor\frac{1+\sqrt{1+4\left(\frac{\mathrm{n}(\mathrm{n}-1)}{2}-\mathrm{q}\right)}}{2}\right\rfloor, \\
& 1 \leq \chi_{\mathrm{dt}}(\mathrm{G}) \cdot \chi_{\mathrm{dt}}(\overline{\mathrm{G}}) \leq\left\lfloor\frac{1+\sqrt{1+4 \mathrm{q}}}{2}\right\rfloor \cdot\left\lfloor\frac{1+\sqrt{1+4\left(\frac{n(n-1)}{2}-\mathrm{q}\right)}}{2}\right\rfloor .
\end{aligned}
$$

Proof. (a) The result is a direct consequence of Lemma 11.

(b) The result is a direct consequence of Theorem 4 .

(c) The result is a direct consequence of Theorem 5 .

Any simple graph $G$ of order $n$ has at most, $\frac{n(n-1)}{2}$ edges. From Theorem 3 it follows that the minimal graph $G$ has order $2 k-1$ and size $k(k-1)$ for $k \in \mathbb{N}$. Hence, $\bar{G}$ has $\varepsilon(\bar{G})=k^{2}-2 k+1$. The aforesaid implies that $\varepsilon(\mathrm{G})-\varepsilon(\overline{\mathrm{G}})=k-1>0, \forall k \geq 2$. Clearly $\mathrm{G}$ is not self-complementary. This leads to a corollary. 
Corollary 19 For the minimal graph $\mathrm{G}$ from Theorem 3 and for $\mathrm{k} \geq 2$ we have, $\chi_{\mathrm{dt}}(\mathrm{G})>\chi_{\mathrm{dt}}(\overline{\mathrm{G}})$.

Proof. Theorem 5 read together with the fact that, $\varepsilon(G)-\varepsilon(\bar{G})=k-1>0$, $\forall k \geq 2$, suffice.

\section{Conclusion}

The degree tolerant chromatic number was introduced subject to conditions (i) and (ii). Different conditions can be formulated to study derivatives of the notion of degree tolerance coloring. For example, for a given $k, 1 \leq k \leq n-1$ a condition such as; if $\operatorname{deg}(u) \leq k$ and $\operatorname{deg}(v) \leq k$ and $u v \in E(G)$ then, $c(u)=c(v)$ else, $c(u) \neq c(v)$ is a derivative for further study.

Problem 1. The procedure to improve the upper bound stated in Lemma 11 follows from condition (i). The fact that applying the procedure exhaustively will yield equality has been stated without proof. Formalise the statement.

Problem 2. Utilise the result in problem 1 to determine exact values of $\chi_{\mathrm{dt}}(\mathrm{G})$ for various classes of graphs.

Problem 3. If possible find improved results for Theorems 16 and 17.

Conjecture. For graphs $\mathrm{G}$ and $\mathrm{H}$ it follows that,

$$
\chi_{d t}(G \times H)=\min \left\{\operatorname{di}(H) \chi_{d t}(G), \operatorname{di}(G) \chi_{d t}(H)\right\}
$$

A worthy avenue for further research would be to consider all known graph products such as, strong product, co-normal product, modular product, rooted product and so on.

It is observed that with regards to the clique number the minimal graph $\mathrm{G}$ constructed in the proof of Theorem 4 is, $\omega(\mathrm{G})=k$. Furthermore, exactly two such induced cliques exist in G. We suggest that studying other graph parameter specific to this minimal graph could be a worthy avenue.

Let $f(k), g(k), h(k)$ be functions such that $f(k)=\min \{g(k), h(k)\}$. If for some $k \in \mathbb{N}$ we have that, $g(k)=h(k)$ then $f(k)$ is said to be tied or equal-valued. If $g(k) \neq h(k)$ then $f(k)$ is said to be non-tied or decisive. A graph of order $\mathrm{n}$ and size $\mathrm{q}$ can be called a $(\mathrm{n}, \mathrm{q})$-graph. It is obvious from Theorems 4 and 5 that the function, $f_{1}(n, q)=\min \left\{\left\lfloor\frac{n+1}{2}\right\rfloor,\left\lfloor\frac{1+\sqrt{1+4 q}}{2}\right\rfloor\right\}$ is tied (equal-valued) for all $(2 k-1, k(k-1))$-graphs.

Problem 4. Prove that $f_{2}(n, q)$ is non-tied (or decisive) for all $(g(k), h(k))$ graphs if the functions, $n=g(k)_{k \in \mathbb{N}} \neq 2 k-1$ or $q=h(k)_{k \in \mathbb{N}} \neq k(k-1)$. 


\section{References}

[1] M. Aouchiche, P. Hansen, A survey of Nordhaus-Gaddum type relations, Discrete Applied Mathematics, 161 (2013) 466-546. $\Rightarrow 229$

[2] J. A. Bondy, U.S.R. Murty, Graph Theory with Applications, Macmillan Press, London, (1976). $\Rightarrow 217$

[3] L. Cowen, R. Cowen, D. Woodall, Defective colorings of graphs in surfaces: partitions into subgraphs of bounded valence, Journal of Graph Theory, 10 (1986) $187-195 . \Rightarrow 217$

[4] L. Cowen, W. Goddard, C. Jesurum, Coloring with defect, Proceedings of the 8th ACM-SIAM Symposium on Discrete Algorithms, 1997, pp. 548-557. $\Rightarrow 217$

[5] F. Harary, Graph Theory, Addison-Wesley, Reading MA, 1969. $\Rightarrow 217$

[6] F. Harary, K. Jones, Conditional colorability II: Bipartite variations, Congressus Numer., 50 (1985) 205-218. $\Rightarrow 217$

[7] N. K. Sudev, On certain $\mathcal{J}$-colouring parameters of graphs, Nat. Acad. Sci. Lett., 43 (2020) 53-57. $\Rightarrow 219$

[8] B. West, Introduction to Graph Theory, Prentice-Hall, Upper Saddle River, (1996). $\Rightarrow 217$

Received: June 9, 2020 • Revised: October 13, 2020 